\title{
Deep sequencing and de novo assembly of the mouse oocyte transcriptome define the contribution of transcription to the DNA methylation landscape
}

\author{
Lenka Veselovska ${ }^{1 \dagger}$, Sebastien A. Smallwood ${ }^{1 *+}$, Heba Saadeh ${ }^{1,2}$, Kathleen R. Stewart ${ }^{1}$, Felix Krueger ${ }^{2}$, \\ Stéphanie Maupetit-Méhouas ${ }^{3}$, Philippe Arnaud ${ }^{3}$, Shin-ichi Tomizawa ${ }^{4}$, Simon Andrews ${ }^{2}$ and Gavin Kelsey ${ }^{1,5^{*}}$
}

\begin{abstract}
Background: Previously, a role was demonstrated for transcription in the acquisition of DNA methylation at imprinted control regions in oocytes. Definition of the oocyte DNA methylome by whole genome approaches revealed that the majority of methylated $\mathrm{CpG}$ islands are intragenic and gene bodies are hypermethylated. Yet, the mechanisms by which transcription regulates DNA methylation in oocytes remain unclear. Here, we systematically test the link between transcription and the methylome.

Results: We perform deep RNA-Seq and de novo transcriptome assembly at different stages of mouse oogenesis. This reveals thousands of novel non-annotated genes, as well as alternative promoters, for approximately $10 \%$ of reference genes expressed in oocytes. In addition, a large fraction of novel promoters coincide with MaLR and ERVK transposable elements. Integration with our transcriptome assembly reveals that transcription correlates accurately with DNA methylation and accounts for approximately 85-90\% of the methylome. We generate a mouse model in which transcription across the Zac1/Plag/1 locus is abrogated in oocytes, resulting in failure of DNA methylation establishment at all CpGs of this locus. ChIP analysis in oocytes reveals H3K4me2 enrichment at the Zac1 imprinted control region when transcription is ablated, establishing a connection between transcription and chromatin remodeling at $\mathrm{CpG}$ islands by histone demethylases.
\end{abstract}

Conclusions: By precisely defining the mouse oocyte transcriptome, this work not only highlights transcription as a cornerstone of DNA methylation establishment in female germ cells, but also provides an important resource for developmental biology research.

\section{Background}

The DNA methylome is particularly dynamic during germ cell specification and gametogenesis, relating to the epigenetic reprogramming required to ensure the renewal of totipotency at each generation [1, 2]. DNA methylation (DNAme) is globally erased during migration of primordial germ cells (PGCs) towards the genital ridge, followed by de novo establishment of new methylation landscapes that are different between male and

\footnotetext{
* Correspondence: sebastien.smallwood@babraham.ac.uk;

gavin.kelsey@babraham.ac.uk

${ }^{\top}$ Equal contributors

'Epigenetics Programme, Babraham Institute, Cambridge, UK

Full list of author information is available at the end of the article
}

female germlines. In females, DNAme is established after birth, during follicular growth, in oocytes in meiotic arrest. Of particular interest, DNAme occurs at a subset of CpG islands (CGIs) termed imprinted germline differentially methylated regions (igDMRs); this gamete-derived methylation is maintained allele-specifically after fertilisation and acts as the basis for regulating genomic imprinting and its hundred mono-allelically expressed genes [3]. The recent development of methods combining bisulfite conversion as a means to determine methylation levels and high-throughput sequencing for low amounts of starting material have allowed the detailed profiling of the DNAme landscapes of germ cells and pre-implantation embryos [4-7]. Notably, these studies have revealed that 
whilst many CGIs are methylated in oocytes, most are not related directly to genomic imprinting but, nevertheless, a significant amount of oocyte-derived DNAme is present in embryonic day (E)3.5 pre-implantation blastocysts $[5,8,9]$. Yet, aside from this descriptive information, the mechanisms by which DNAme is established and regulated in oocytes, and its biological function aside from genomic imprinting, are still largely unclear.

Acquisition of DNAme at a genomic locus is likely to require integration of a combination of several factors, such as DNA sequence, specific trans-acting factors, and cross-talk between histone modifications and DNA methyltransferases (DNMTs) [2, 10]. Focusing on the Gnas imprinted locus, we established a functional link between transcription across an igDMR from an upstream transcription start site (TSS) and establishment of DNAme during oogenesis [11]. Similar results were subsequently obtained by others, as well as for the Snrpn imprinted locus [12, 13]. In addition, by performing reduced representation bisulfite sequencing (RRBS) in mature oocytes, we found that methylated CGIs are preferentially located within transcription units, highlighting a potential global role for transcription in determining the DNAme landscape of female germ cells [5]. This conclusion later received support when the first whole genome DNA methylome of these cells was reported, with evidence that gene bodies were enriched in DNAme [4]. The mechanistic role for transcription in DNAme establishment is likely (at least in part) to be a consequence of how the targeting of DNMT3A, and its co-factor DNMT3L, is regulated by histone post-translational modifications. Indeed, while histone 3 lysine 4 (H3K4) methylation has been shown to inhibit interaction of DNMT3A and DNMT3L with nucleosomes, H3K36me3 (a transcription elongation mark) enhances DNMT3A activity [14, 15]. These properties of the de novo methylation complex suggest that transcription could account for the majority of the oocyte methylome. Yet to what extent transcription controls DNAme establishment is undetermined and represents an unresolved question towards a full understanding of epigenetic reprogramming during development.

Regulation of transcription in oocytes is unique because of the distinctive nature and biological roles of these cells. They are highly transcriptionally active prior to and during the establishment of DNAme - with abundant accumulation of transcripts - and transcriptionally silent when mature. These transcripts serve not only to control oogenesis but also as a "maternal pool" for the regulation of pre-implantation development until zygotic and mid-preimplantation embryonic gene activation [16]. While our knowledge of the mouse oocyte transcriptome has greatly improved in recent years due to the development of RNA sequencing (RNA-Seq) for low amounts of input $[4,5,16-18]$, such studies are limited because they relied on the annotated reference genome as a basis for their analysis, leading de facto to a loss of potentially critical information. Indeed, we have, for example, revealed that expression of imprinted genes in oocytes can be controlled by non-annotated oocytespecific TSSs, and multiple studies in pluripotent and somatic cells have revealed the existence of non-coding RNAs (ncRNAs) which are not indexed in reference annotations [11, 19]. Therefore, to properly evaluate the contribution that transcription makes to patterning the oocyte methylome, a comprehensive description of the oocyte transcriptome and promoter use is required.

In this study, we set out to define precisely the correlation between transcription and the DNAme landscape in the following integrated approach. We sought to generate a high-quality transcriptome annotation by deep RNA-Seq of oocytes during follicular growth at the time of active de novo DNAme, with a particular focus on the identification and characterization of novel genes and TSSs; this analysis revealed a key role for transposable element (TE) expression in determining oocyte-specific transcription events. From nucleotide-resolution maps, we analysed the distribution of DNAme in the oocyte, and determined that the genome is partitioned into large-scale hypermethylated and hypomethylated domains, a distinctive feature of the oocyte methylome. By integrating these datasets, we assessed the coincidence of transcription units with hypermethylated domains. By this analysis, transcription accounts for up to $90 \%$ of the methylome, but there are also exceptions to a simple, transcription-dependent model. Finally, we functionally demonstrated the requirement of transcription in establishing DNAme at all CpGs of a locus using transgenic mice.

\section{Results and discussion}

\section{Our deep RNA sequencing approach outclasses previously} published datasets

Several limitations were present in the datasets published by us and others prior to and during the course of this project, irrespective of their overall low sequencing depth $[4,5,16-18,20,21]$. First, apart from one study [16], only the poly-adenylated (poly(A))-enriched fraction was sequenced, while much evidence demonstrates the existence of long non-poly(A) transcripts transcribed by RNA polymerase II in mammalian cells [22]. Second, these data were mostly not strand-specific (i.e., there was no information on transcription orientation), hence limiting the accurate identification of alternative TSSs, for example. Finally, the datasets were generated from transcriptionally silent fully grown germinal vesicle and metaphase II oocytes, after DNAme establishment, and therefore potentially lacked transcripts expressed during 
early oocyte growth but degraded before the completion of oocyte development.

To circumvent these limitations, we generated strandspecific RNA-Seq libraries using ribosomal RNA depletion on oocytes isolated at different stages of follicular growth (i.e., non-growing oocytes (NGOs); growing oocytes (GOs; GO1 for mice aged 8-14 days post-partum (dpp), GO2 for mice aged $15 \mathrm{dpp}$ ); fully grown oocytes (FGOs)) (Table 1; Fig. 1a). Libraries were sequenced with 100 base pair (bp) paired-end reads, with a total number of reads generated of $\sim 280$ million, of which $\sim 190$ million were concordant paired-end reads. This resulted in a total of 129.7 Mbp covered by at least five unique reads, $80.7 \mathrm{Mbp}$ of which were located outside the reference genome annotation (merging of Ensembl, University of California, Santa Cruz (UCSC) and RefSeq non-redundant transcripts isoforms). This represented an increase of $203.5 \%$ over all the previously published datasets merged together (63.7 Mbp, 74.0 Mbp outside reference) (Fig. 1b; Figure S1a in Additional file 1). In addition, we reliably identified (covered by at least five unique reads) 283,171 splice junctions/exon boundaries matching the reference annotation and 74,037 novel ones, representing again a significant increase over the published datasets (258,033 and 33,782, respectively) (Fig. 1c; Figure S1a in Additional file 1).

\section{Definition of the mouse oocyte transcriptome}

To generate our oocyte reference annotation, we used Cufflinks, a genome-guided transcriptome assembler, using a combination of strategies [23, 24]. We performed Cufflinks on all datasets individually or merged, in default genome-guided mode or in reference annotationbased transcript (RABT) mode [25]; this combination of strategies was used because it was found that the different options tested gave different results, necessitating a composite approach for accurate assembly. Non-transcribed reference annotated transcripts included in the RABT mode (53-64 \% total transcripts) were removed based on their expression values (Figure S1b in Additional file 1). All different assembly annotations were then merged into a single oocyte transcriptome annotation using the program Cuffmerge (Fig. 1a). It is known that Cufflinks can generate artefacts [26], and the raw oocyte annotation had to be curated step-by-step to remove new transcripts for which true biological identity was contentious (Fig. 1a; Figure S1c in Additional file 1; "Materials and methods").

The final oocyte transcriptome annotation consists of 82,939 transcripts forming 39,099 expressed genes (14,995 mono-exonic and 24,104 multi-exonic), where a gene is a transcription unit that may comprise multiple transcript isoforms. Out of these, 20,428 genes (52.3\%), representing 6877 multi- and 13,551 mono-exonic genes, were only found in our oocyte annotation, the rest corresponding to known or novel isoforms of transcripts present in the reference annotation (Fig. 1d; Figure S1d in Additional file 1; Table S1 in Additional file 2). However, some of these novel transcripts may still correspond to known ncRNAs not present in the reference, as well as incomplete annotations of extended known transcripts. Therefore, for higher confidence in the identification of genuinely novel genes, we excluded all genes overlapping or in close proximity, on the same strand, to reference genes or known ncRNAs from the NONCODEv4 database $( \pm 1 \mathrm{kbp}$ and $\pm 5 \mathrm{kbp}$ for multi- and mono-exonic genes, respectively) [27]. Furthermore, mono-exonic genes representing expressed independent repetitive elements annotated in RepeatMasker were excluded. This strategy resulted in the higher confidence identification of 3848 novel multi-exonic genes and 5165 novel mono-exonic genes ( $23.1 \%$ of total; 13,809 transcripts; Fig. 1d). It should be noted that using these empirical criteria could have led to the removal of true biological transcripts, and some artefacts may remain in our final annotation. We have tested multiple parameters of analysis, and we believe the approach presented here was the most stringent possible and is fully adequate for the characterization and analysis performed below.

\section{A fraction of novel oocyte transcripts are potentially coding} To validate our experimental approach, we examined how many novel oocyte transcripts defined by our transcriptome assembly could retrospectively be identified using the previously published oocyte datasets. We observed that $94.3 \%$ of novel multi-exonic and $55.1 \%$ of novel mono-exonic genes are detected in these datasets merged together (FPKM (fragments per kilobase of transcript per million mapped reads) $>0.008$, defined using

Table 1 RNA-Seq samples and sequencing characteristics

\begin{tabular}{|c|c|c|c|c|c|c|}
\hline Sample ID & Type & Age of mice (dpp) & Oocyte size & Number of oocytes & Raw sequencing reads & Uniquely mapped reads \\
\hline $\mathrm{NGO}$ & Non-growing oocytes & 5 & $10-40 \mu \mathrm{m}$ & 1545 & $44,111,934$ & $31,173,658$ \\
\hline GO1 & Growing oocytes & $8-14$ & $25-70 \mu \mathrm{m}$ & 1990 & $67,882,721$ & $42,709,253$ \\
\hline GO2 & Growing oocytes & 15 & 50-70 $\mu \mathrm{m}$ & 1510 & $118,463,451$ & $82,037,819$ \\
\hline \multirow[t]{2}{*}{ FGO } & Fully grown oocytes & 20 & $>70 \mu \mathrm{m}$ & 832 & $47,855,997$ & $35,107,487$ \\
\hline & & & Total & 5877 & $278,314,103$ & $191,028,217$ \\
\hline
\end{tabular}




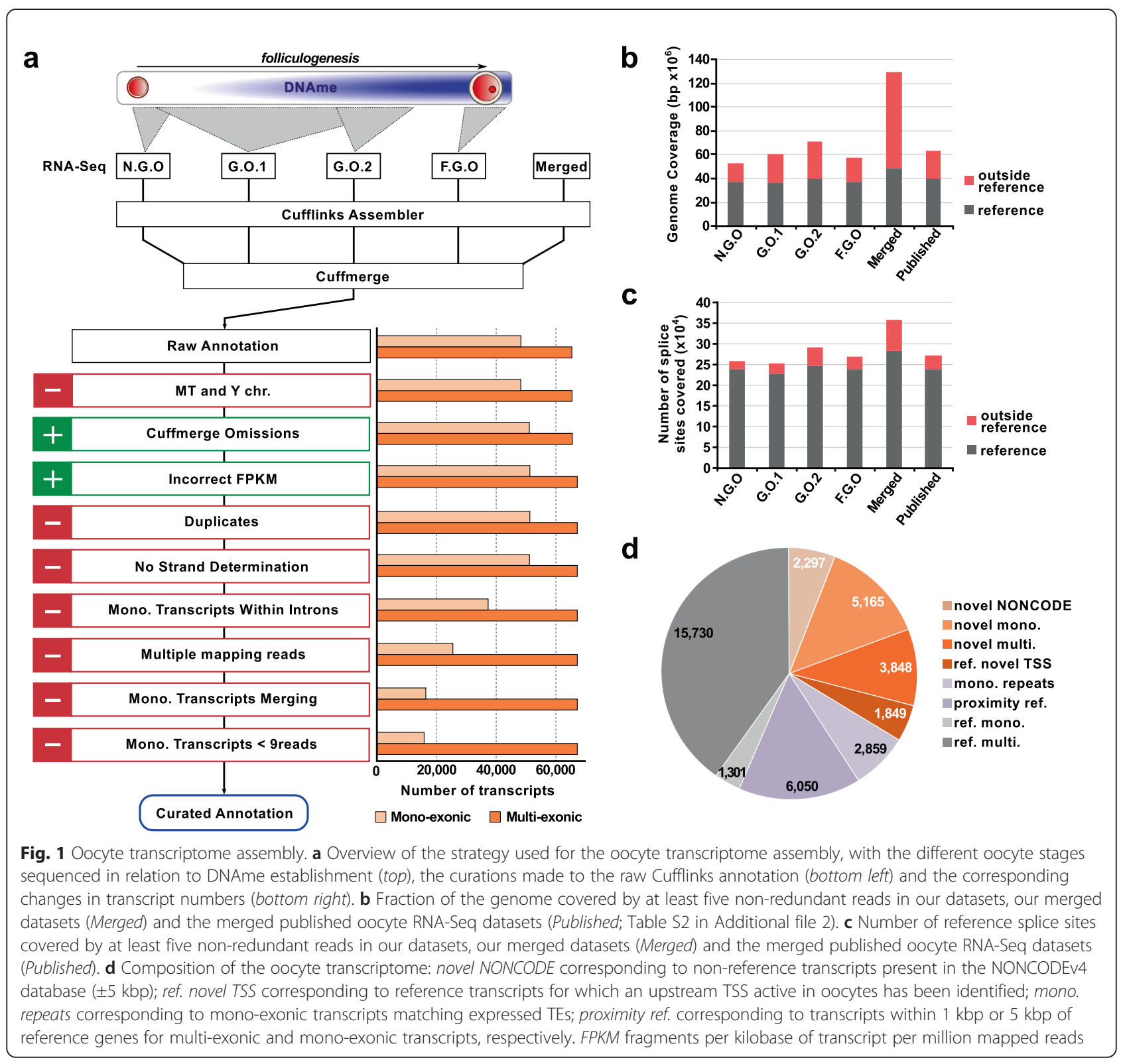

the same approach as in Figure S1b in Additional file 1), and logically the overlap is greater for more highly expressed genes (Figure S2a in Additional file 1). We also validated by RT-PCR a random selection of novel genes (14) with a $100 \%$ success rate for both multi- and mono-exonic genes (Figure S2b in Additional file 1).

While novel genes represent $23.1 \%$ of all expressed genes in our oocyte transcriptome, they are, on average, shorter than reference genes (median of $2.5 \mathrm{kbp}$ and $19.1 \mathrm{kbp}$, respectively) and represent only $7.6 \%$ of the genomic fraction occupied by all expressed genes. In addition, the expression level of reference genes is substantially higher than that of novel genes (median FPKM of 2.65 and 0.19 , respectively, from GO2 oocytes; Fig. 2a).
It is legitimate to assess what proportion of the novel oocyte genes is likely to have biological function. Therefore, we tested the potential of novel transcripts to encode proteins through the use of the specialised programs Coding Potential Calculator (CPC) and CodingNon-Coding Index (CNCI) [28, 29]. CPC identified 841 mono- and 834 multi-exonic genes $(18.6 \%$ of all novel genes) and CNCI 100 mono- and 188 multi-exonic genes $(3.2 \%)$ as having coding potential. Even if predictions based on such bioinformatic tools must be considered with care (the overlap between $\mathrm{CPC}$ and $\mathrm{CNCI}$ is small -171 genes), this suggests that we have identified a substantial number of ncRNAs. Interestingly, novel genes that appear to be oocyte-specific as they are not 


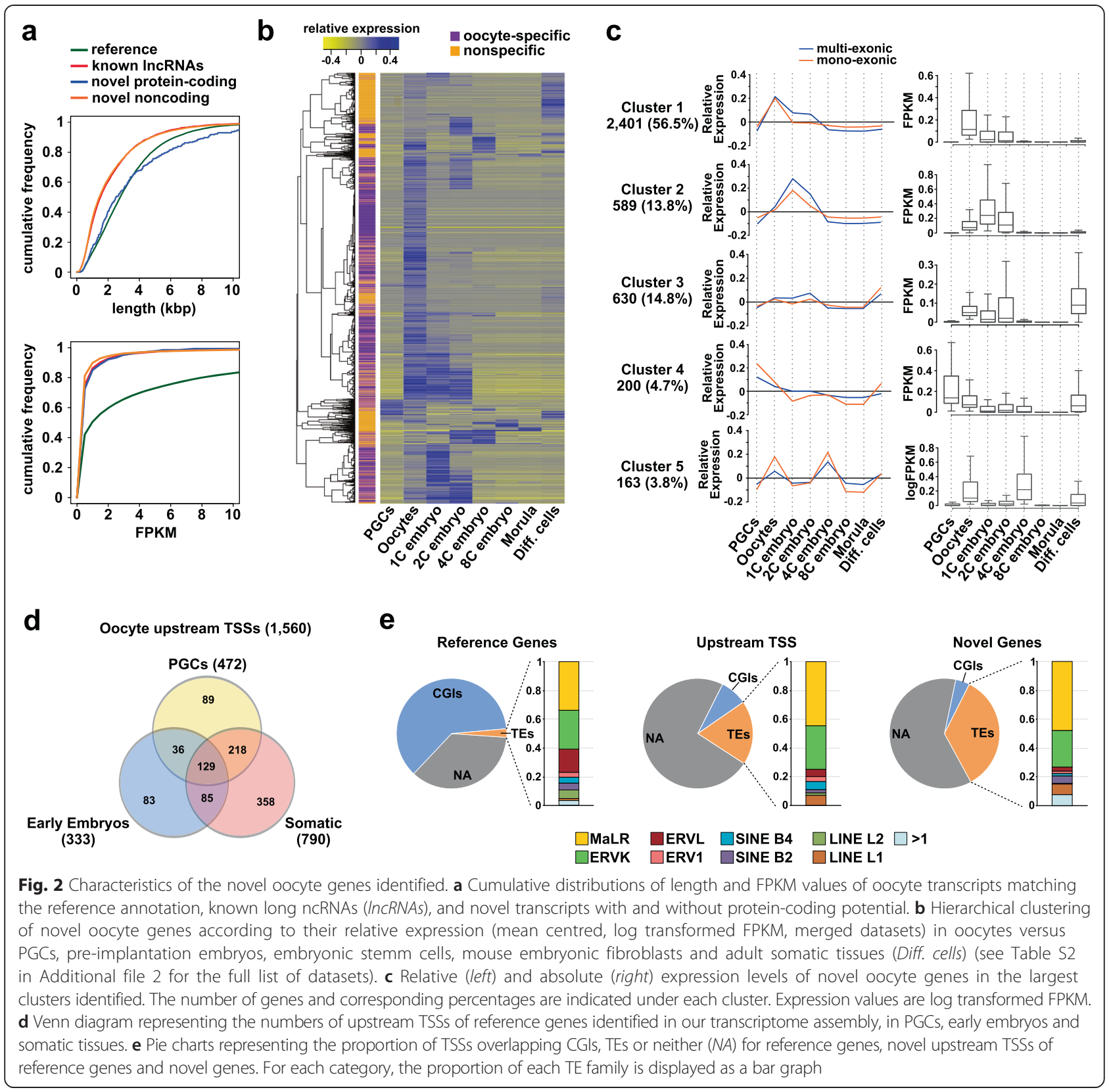

detected beyond the four-cell stage ( $\sim 56 \%$; see below and Fig. 2b, c) are more likely to be non-coding than the novel genes detected in other developmental stages or in somatic tissues (49 versus 122 genes with coding potential based on both CPC and CNCI, respectively). Focusing on the protein domains of these coding-potential genes using blastx tool hits to the Uniprot database [30], we observed that at least nine novel genes possess known protein domains. Of interest, these include the Hop1p, Rev7p, and MAD2 (HORMA) domain, a chromatin-binding domain found in proteins regulating meiotic chromosome behaviour and DNA repair during meiosis. Interestingly, known proteins with
HORMA domains have been demonstrated to play key roles in oogenesis [31].

\section{Identification of novel oocyte transcripts specifically expressed in female germ cells}

If transcription patterns the DNA methylome of the oocyte, it is interesting to assess how many such transcription events are unique to the oocyte. To determine the fraction of novel transcripts that are specifically expressed in oocytes, we investigated their expression profiles in PGCs, throughout pre-implantation embryonic development (zygote to morula), in embryonic stem cells (ESCs) and various somatic tissues using relevant publicly 
available datasets (Table S2 in Additional file 2). Since most of these datasets are not strand-specific, we only analysed novel transcripts that do not overlap with others (2221 multi-exonic and 3210 mono-exonic genes). We performed hierarchical clustering analysis for novel genes expressed in at least one developmental stage (FPKM $\geq$ 0.1; 2075 multi-exonic and 2188 mono-exonic genes; Fig. 2b, c; Figure S2c in Additional file 1) and we observed that $\sim 56 \%$ of novel genes were classified as potentially oocyte-specific $(54.1 \%$ of multi- and $58.1 \%$ of monoexonic genes; principally clusters 1 and 2; Fig. 2c) based on their expression being detected in oocytes and up to four-cell embryos only, in accordance with a recent study examining timing of degradation of maternally provided transcripts after fertilisation [16] (Fig. 2b, c). Focusing on the PGC:oocyte transition, we determined that only 13.2 $\%$ of novel genes appear to be expressed already in PGCs (principally cluster 4), suggesting a profound remodelling of the transcriptome during specification of oocytes. However, it should be noted that inaccuracies could potentially arise from comparing datasets generated by different methods, and we cannot exclude at this stage that some of the novel oocyte genes are expressed at low levels at other developmental stages but are not detected in the respective datasets analysed.

\section{Characterization of novel transcription start sites reveals the contribution of transposable elements to the oocyte transcriptome}

Previous results from our laboratory highlighted, in the context of genomic imprinting, the existence of alternative TSSs in oocytes non-annotated in the genome reference [11]. To investigate this genome-wide, we focused on genes for which TSSs are located in separate novel exons and outside reference TSS-associated CGIs. Using these criteria, we identified new upstream promoters active in oocytes for 1849 multi-exonic reference genes (10.8 \% total expressed; Fig. 1d). Of note, the median distance between the reference and novel TSS was 5.3 kbp. Similar to novel genes, $79.9 \%$ of these novel TSSs can be retrospectively classified as expressed/active in published oocyte datasets, and RT-PCR assays confirmed the expression of nine out of twelve randomly selected novel TSSs (this incomplete success could be attributed to limitation in primer design and sensitivity of detection in material of limiting availability; Figure S3a, b in Additional file 1). Interestingly, novel upstream TSSs of reference genes are less often located within CGIs compared with reference-annotated TSSs (8.7 \% versus $49.4 \%$, respectively). This is similar to all novel transcripts identified in our oocyte annotation, with only $4.6 \%$ (410) having a CGI-associated TSS (62\% for CGI-associated TSSs of reference genes expressed in the oocytes) (Fig. 2d).
By measuring the activity of the novel upstream TSSs of reference genes in other developmental stages, we found that $35.7 \%$ appear to be oocyte-specific, as they were not detected in PGCs, eight-cell embryos, morula or any of the other cell types examined (1560 analysed genes with TSSs not overlapping with other genes) (Fig. 2e; Figure S3f in Additional file 1). Importantly, only $30.3 \%$ of all novel upstream TSSs were detected in PGCs, highlighting again the substantial remodelling of the transcriptome associated with oocyte specification. Classifying genes based on their expression from upstream or reference TSSs shows that the most common pattern is that the gene is expressed from the upstream TSS in oocytes, but from the reference TSS in PGCs, embryos and differentiated cells (Figure S3f in Additional file 1).

Next, we aimed to identify common features for the novel TSSs active in oocytes (novel transcripts plus alternative TSSs of reference genes). A particularity of oocytes is the high transcriptional activity of TEs, and it was reported that TEs could act as promoters for a limited number of transcripts in mouse oocytes and ESCs $[17,32,33]$. To investigate this further, we first quantified the expression of TEs in our oocyte datasets. This revealed that the ERVK and especially MaLR families from the long terminal repeat class are highly expressed, in accordance with previous observations $[33,34]$ (Figure S3c in Additional file 1). Importantly, we found that TE-associated TSSs are found in 34.6 \% (3121) of novel genes, and in 20.4 \% (377) of novel upstream TSSs of reference genes; this is significantly higher than for annotated TSSs of expressed reference genes $(478 ; 2.5 \%)$. However, and of particular interest, only MaLR and ERVK elements act as TSSs more often than expected by chance based on occupancy of intergenic regions by individual TE families, with 282 novel upstream TSSs of reference genes and 2607 TSSs of novel genes coinciding with these TEs (Fig. 2d; Figure S2d in Additional file 1). Of note, the expression of novel genes with MaLR- and ERVK-associated TSSs (median FPKM values 0.259 and 0.325 , respectively) is higher than novel genes with TSSs in unique sequences (median FPKM value 0.168, GO2 dataset) (Figure S3e in Additional file 1). In addition, ERVK and MaLR elements associated with promoters of novel genes are hypomethylated (18.3\% and $8.7 \%$, respectively) compared with the genome average (36.8 \% and $33.4 \%$, respectively) and intergenic regions $(28.0 \%$ and $17.1 \%$, respectively).

\section{The oocyte DNA methylome is composed of large-scale} hypermethylated and hypomethylated domains Previous studies based on whole-genome bisulfite sequencing revealed that the global DNAme level in fully grown germinal vesicle oocytes is approximately 
$40 \%$ [4, 35], with a strongly bimodal distribution of methylation of CpGs, in contrast to what is observed in sperm, ESCs and typical somatic tissues. By examining in detail the oocyte DNA methylome, we observed that methylated and unmethylated CpGs are not distributed randomly throughout the genome. Instead, analysis of DNAme levels of consecutive $1 \mathrm{kbp}$ genomic windows revealed that methylated $\mathrm{CpGs}$ tend to cluster together, such that the DNA methylome is composed of large-scale hypermethylated domains (HyperD) and hypomethylated domains (HypoD) (Fig. 3a, b).

To assess the extent of the DNA methylome that depends on transcription, we defined HyperDs and HypoDs bioinformatically. For this, as described in detail in the "Materials and methods" section, we determined the methylation level of genomic intervals corresponding to 50 consecutive CpGs (median size of $5.4 \mathrm{kbp}$ ), with overlapping steps of ten consecutive CpGs. We then merged consecutive windows with similar methylation levels, using cutoffs of 75-100 \% and 0-25 \% for HyperDs and HypoDs, respectively. For better correlation between transcription and DNAme (see below), we excluded from the domain definitions genomic regions corresponding to promoters and CGIs, as these features are likely to be unmethylated and would split one HyperD into several HyperDs but still potentially associated with one transcriptional unit (the correlation between transcription and CGI methylation is investigated separately below). We also excluded regions with $50 \%$ or higher methylation in DNMT3A- or DNMT3L-deficient oocytes or early NGOs, as it was not possible to conclude how much of the ultimate DNAme in these regions could be a result of de novo events (Figure S4a, b in Additional file 1). Of note, all the analyses listed below were also performed using domains defined with genomic windows of ten consecutive CpGs with five consecutive CpG steps (median size 940 bp); the results being essentially the same, we describe results only for the former (50/10) conditions for clarity.

Our experimental approach resulted in the definition of 21,044 HyperDs and 25,165 HypoDs (46,209 domains in total; Additional file 4). Importantly, the majority of genomic CpGs are represented within HyperDs and HypoDs with $30.7 \%$ and $50.7 \%$ total CpGs, respectively, the remaining corresponding principally to promoters and CGIs (11.2\%) and regions with intermediate levels of methylation in FGOs (5.5 \% total CpGs, 25-75\% methylation level) (Fig. 3c). As expected, $90.0 \%$ of CpGs in HyperDs are methylated $(\geq 75 \%$ DNAme, average methylation $91.4 \%$ ) and $89.3 \%$ of CpGs in HypoDs are unmethylated ( $\leq 25 \%$ DNAme, average methylation $8.3 \%$ ), validating the accuracy of our approach and the concept of large-scale domains (Fig. 3d). HyperDs appeared smaller with an average size of $35.9 \mathrm{kbp}$ (median $20.9 \mathrm{kbp}$ ) compared with $59.2 \mathrm{kbp}$ (median $24.9 \mathrm{kbp}$ ) for
HypoDs, reflecting the overall genome methylation (40\%) (Fig. 3e). Of note, the average CpG density was similar for HyperDs and HypoDs (1.8 and 1.7, respectively; Fig. 3e).

\section{Hypermethylated domains overlap with active transcription} units

In order to test the strength of the association between transcription and DNAme, we quantified the proportion of each domain overlapped by transcription events. Based on our oocyte transcriptome annotation, $74.3 \%$ of HyperDs are overlapped by transcripts for at least $50 \%$ of their length (63.2 \% for $80 \%$ of their length; Fig. 3f). Since our oocyte transcriptome assembly was very stringent and the Cufflinks assembly could have missed some transcripts, we also defined transcribed units as regions with a minimum of three overlapping reads in at least one of our oocyte RNA-Seq datasets. Based on this, we found that $79.8 \%$ of HyperDs are associated with transcription events ( $>50 \%$ of the domain overlapped by a transcription unit; Fig. 3f). When considering the total length of all HyperDs together, $88.8 \%$ overlaps with transcription units. Logically the correlation with transcription is dependent on the size of the HyperD, but interestingly the overlap with transcription units is higher for large domains (91.1\% of HyperDs $>50 \mathrm{kbp}$ are overlapped by transcription units for $>50 \%$ of the domain, which is $20.9 \%$ of all HyperDs) compared with small domains $(71.9 \%$ of HyperDs $<10 \mathrm{kbp}-22.2 \%$ of all HyperDs), suggesting that some short domains in particular may require additional mechanisms for their DNAme establishment (Figure S5a, b in Additional file 1).

Despite the strong association with transcription, our analysis revealed that 2052 HyperDs (9.8 \% of total) and some parts of HyperDs do not appear to be associated with transcription events $(<5 \%$ of their length overlaps transcribed regions). Of note, these apparently nontranscribed HyperDs are relatively short compared with all HyperDs, with an average size of $17.5 \mathrm{kbp}$ (median $13.1 \mathrm{kbp}$ ). We set out to identify other features of these HyperDs that could contribute to DNAme establishment. We found that, compared with transcriptionally silent HypoDs, these HyperDs are enriched in ERVK elements, and also in intermediate levels of methylation (25-50\%) in NGOs or DNMT3A- and DNMT3Ldeficient oocytes. However, these features represent only $1.7 \%$ of the total length of all HyperDs. Nevertheless, when considered with transcription, this revealed that only $9.5 \%$ of the combined length of HyperDs is unaccounted for (Figure S5b in Additional file 1). Evidently, it could be that our RNA-Seq strategy failed to capture some transcription events. This is difficult to assess, but seems less likely for highly transcribed regions, and lowly transcribed regions are usually hypomethylated 


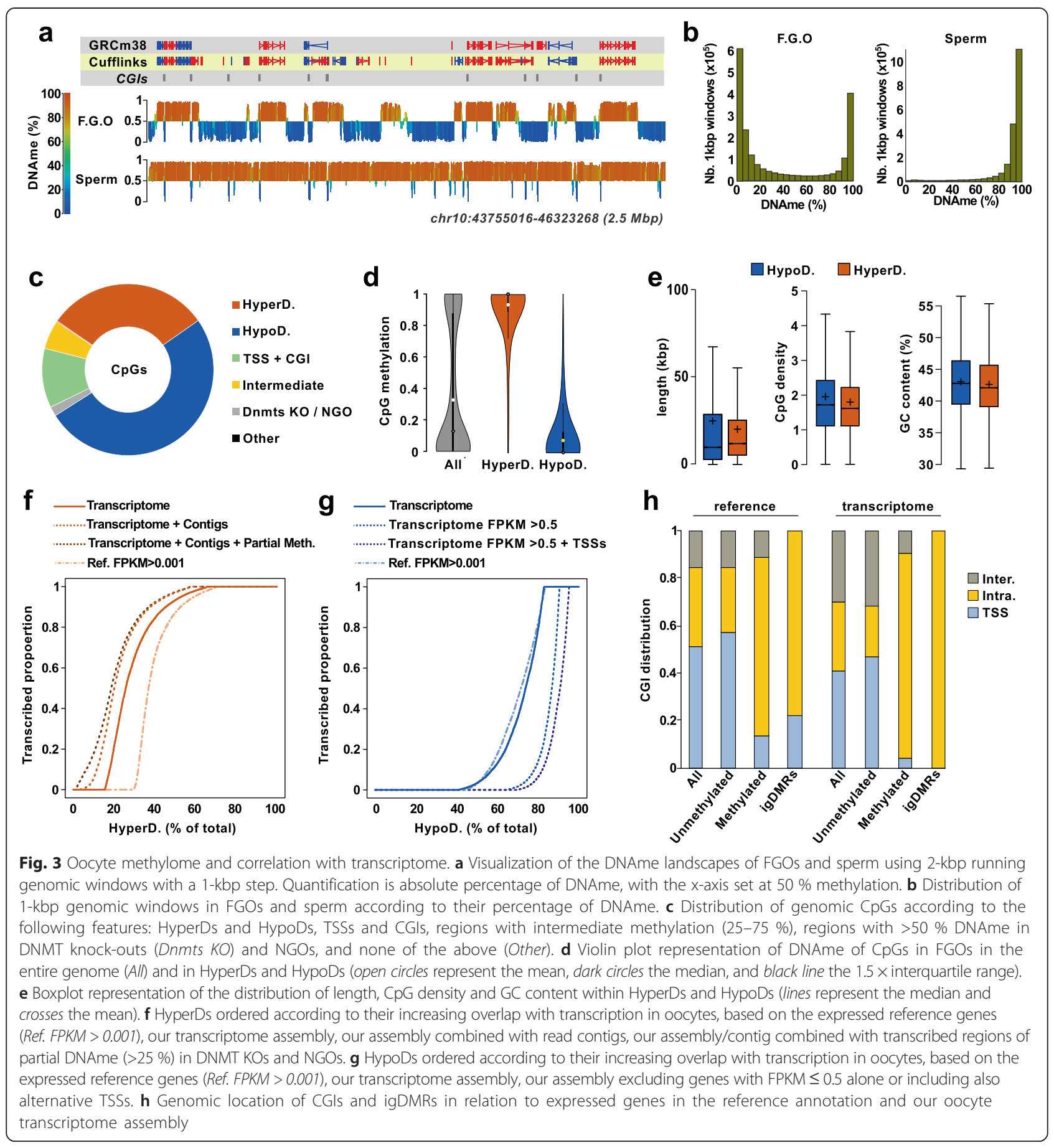

(see below). Another explanation for this could relate to DNAme spreading, as observed in different contexts such as TEs, but this remains controversial and has not been shown in a germ cell context [36]. Alternatively, a transcription-independent mechanism could exist, based possibly on the interaction of DNMT3A/3L with specific histone marks other than H3K36me3. Further development of ChIP-Seq protocols for low amounts of starting material would be necessary to investigate this.
Having found a significant proportion of novel transcripts identified by our deep RNA-Seq approach, we investigated in more detail how this class contributes to the DNAme landscape. Our oocyte transcriptome contains $83.0 \%$ of the methylated CpGs versus $75.3 \%$ for the reference annotation: $4.5 \%$ of methylated CpGs are within new genes. Focusing on transcripts expressed from TEs as promoters, for both novel genes and alternative upstream TSSs, a direct association was found for $4.7 \%$ 
of methylated CpGs in oocytes. Similarly, novel genes and TE-regulated transcripts account for $2.9 \%$ and $5.7 \%$ of methylated CGIs, respectively.

\section{Some expressed genes escape DNA methylation}

If transcription were the predominant factor in determining DNAme in oocytes, it would be surprising to find active transcription units devoid of DNAme. Therefore, we investigated HypoDs for which our oocyte annotation revealed substantial overlap with transcription. These correspond to $26.2 \%$ of all HypoDs (overlap of $>50 \%$ with transcription units), or $16.1 \%$ of the total length of HypoDs, which is quite a significant proportion (Fig. 3g; Figure S5c in Additional file 1). Of note, transcribed HypoDs are relatively small (median length $9.4 \mathrm{kbp}$ ), with $51.9 \%$ and $23.3 \%$ of these domains shorter than $10 \mathrm{kbp}$ and $5 \mathrm{kbp}$, respectively. This size consideration could explain why they escape de novo methylation since short genes typically have low enrichment in H3K36me3 irrespective of expression level [37].

We found that long transcribed HypoDs are frequently associated with genes with very low FPKM values and, in accordance with previous observations, we observed that gene body DNAme levels are positively correlated with transcription levels, likely reflecting degree of H3K36me3 enrichment [4]; indeed, genes with $<0.5$ FPKM are more often unmethylated than methylated, while the proportion of methylated genes increases with increasing FPKM value (Figure S5e in Additional file 1). We found that $46.2 \%$ of transcribed HypoDs (median length $14.7 \mathrm{kbp}$, accounting for $11.0 \%$ of the total length of HypoDs) are associated with genes with $<0.5$ FPKM. In addition, we found that some of the HypoDs defined (14.2\%; 972) correspond to alternative downstream promoters active in oocytes according to our transcriptome assembly; these are shorter on average (median length $4.4 \mathrm{kbp}$ ) and could be protected from de novo methylation by H3K4me2/me3 marks [15] (Fig. 3g; Figure S5c in Additional file 1). Taking into consideration our transcription-based model for de novo DNA methylation, $9.2 \%$ of all HypoDs (3.7\% of total length of HypoDs) appear to be transcribed ( $>50 \%$ overlap) but their methylation status is not directly explained (Figure S5d in Additional file 1).

This prompted us to investigate how many expressed genes escape DNAme. We first identified 318 genes with gene-body DNAme $<25 \%$, but with characteristics of normally methylated genes (FPKM $>1$ and at least 10 $\mathrm{kbp}$ in size). To examine this further, we generated contigs (at least three mapped reads) for each dataset and analysed the methylation level of each gene using a running window strategy. This approach was used to limit potential Cufflinks artefacts, where only a fraction of the wrongly annotated gene would actually be transcribed and methylated. This confirmed 52 large and highly expressed genes (41 genes present in the reference annotation) as unmethylated throughout their entire gene body, and therefore in contradiction to our transcriptionbased model (Table S3 in Additional file 2). Of note, these genes are expressed at high levels throughout folliculogenesis, prior to and after the onset of DNAme targeting. Although gene ontology analysis failed to report significant enrichment for the 41 reference genes, it nevertheless regrouped genes important for meiosis and germ cell development (Sohlh2, Slit3, Syce1, Tes), known transcription regulators (Foxo6, Zbtb38, and Zfp219), as well as members of the Sox and Pax families (Sox13, Pax6).

\section{Transcription and DNA methylation establishment at CGls and igDMRs}

Having demonstrated the substantial contribution of transcription to the global DNA methylome, we next focused on specific genomic features: CGIs. Our oocyte annotation redefined CGI location compared with the reference annotation, and these can be divided into four groups: 9439 CGIs associated with the most upstream TSS of the gene (41.0 \% of total); 1666 CGIs intragenic but associated with downstream/alternative TSSs of the gene (7.2\% of total); 5043 CGIs intragenic and not overlapping a TSS (21.9\% of total); 6861 intergenic CGIs ( $29.8 \%$ of total). Of relevance, and highlighting the benefits of our transcriptome assembly approach, we found that $18.6 \%$ of intergenic CGIs according to the reference annotation are associated with genes in oocytes, and $13.6 \%$ of CGIs originally classified as the most upstream TSS are found to be intragenic.

Based on whole-genome bisulfite sequencing data in FGOs, 2047 CGIs were found to be hypermethylated ( $\geq 75 \%$; $9.1 \%$ total CGIs) and 19,547 hypomethylated ( $\leq 25 \%$; $87.1 \%$ total CGIs). We found that $86.5 \%$ (1771) of the methylated CGIs are located within transcription units, while $3.8 \%$ (78) are associated with the most upstream TSS and $9.7 \%$ (198) are intergenic (Fig. 3h). Of note, $47.9 \%$ (135) of the methylated CGIs overlapping the most upstream TSSs in the reference annotation become intragenic in our oocyte transcriptome. This results either from the existence of alternative upstream TSSs, or from new overlapping transcripts that are in $\sim 25 \%$ of the cases transcribed in the antisense orientation and regulated by a promoter located downstream of the methylated CGI.

Looking in more detail into the exceptions to a transcription-based mode strictly based on our Cufflinks assembly, we found a large fraction of intergenic CGIs (48\%) were still overlapped by transcribed units defined as regions with at least three overlapping reads in at least one of the oocyte RNA-Seq datasets; this was the case for only $15.7 \%$ of unmethylated intergenic CGIs 
(Chi-squared test, $p$ value $<0.0001$ ). Similarly, we observed a tendency for DNAme to extend beyond the 3' end of a gene (for the top $40 \%$ of genes based on their expression, DNAme is still above $75 \%$ at $1 \mathrm{~kb}$ downstream) and $18.7 \%$ of methylated intergenic CGIs overlap with the first $1 \mathrm{kbp}$ downstream of a gene. For the remaining TSSs and intergenic CGIs, we investigated their methylation level in NGOs, oocytes deficient in DNMT3A and DNMT3L, and sperm, but found less than ten to be methylated in these cases.

We next asked whether all CGIs located within transcription units acquire DNAme, as might be predicted from a transcription-based model. Out of the 2863 intragenic unmethylated CGIs, $41.5 \%$ are in close proximity (within $2 \mathrm{kbp}$ ) of the most upstream TSS, or overlapping, or in close proximity to a "downstream" alternative TSS, which might preclude their de novo methylation on the basis of spread of H3K4 methylation. In addition, $41.5 \%$ of intragenic unmethylated CGIs are embedded within larger hypomethylated domains, mostly located within weakly transcribed gene bodies that do not support DNAme establishment. Ultimately we found only 136 CGIs unmethylated but located within a highly transcribed unit and surrounded by a hypermethylated domain. In this case, their methylation state could relate to general mechanisms protecting against DNAme at these genomic elements, and their capability to adopt specific chromatin signatures solely based on their GC-rich sequence [38, 39]; further improvement in ChIP-Seq methodologies will allow this possibility to be investigated in more detail. In conclusion, we found that the transcriptome not only defines a large fraction of methylated CGIs, but could also account for the hypomethylated state of the majority of CGIs.

Having shown that transcription correlates with CGI methylation, we focused on the specific subclass of these genomic features: igDMRs. Based on the reference annotation, 5 out of 23 maternal igDMRs overlap promoter regions (Peg10, Peg3, Slc38a4, AK008011, and Impact), the remainder being within annotated transcription units. Our transcriptome assembly now allows us to identify novel upstream TSSs for the Peg10, Peg3, and Impact genes, and novel transcripts transcribing through the AK008011 and Slc38a4 igDMRs (Fig. 3h; Figure S6 in Additional file 1). A recent publication identified 11 new putative maternal igDMRs [7], and our transcriptome revealed an intragenic location for nine of them. For the remaining two, AK086712 and Pvt1, the associated igDMRs appear to be intergenic according our transcriptome, but are nevertheless located with HyperDs. These results highlight that transcription is the only common feature of maternal igDMRs, to our knowledge, and could link oocyte-specific signalling pathways to the establishment of genomic imprinting.

\section{Transcription is functionally required for DNAme establishment at the Zac1 locus}

Using a mouse model we originally provided a functional demonstration of the importance of transcription in the establishment of DNAme at the igDMRs of the Gnas locus [11]. For technical reasons, however, the poly(A) cassette strategy we used to block transcription was not fully efficient, resulting in variable loss of methylation between mice and precluding the use of this model for more refined and mechanistic analysis. In addition the Gnas locus is particularly complex with multiple igDMRs controlling expression of multiple transcripts (including antisense). For these reasons, we decided to test in more detail the role of transcription in DNAme targeting at another, more tractable locus.

We decided to focus on the imprinted gene Zac1 (Plagl1) principally because of the simplicity of the locus (only one imprinted gene, with igDMR overlapping the annotated canonical promoter), and because a human imprinted disorder is associated with ZAC1 igDMR loss of methylation (transient neonatal diabetes mellitus) [40]. We previously identified by $5^{\prime}$ RACE (rapid amplification of $5^{\prime}$ complementary DNA ends) an oocyte alternative TSS, located $\sim 30 \mathrm{~kb}$ upstream of the Zac1 promoter (which is not active in oocytes), regulating the expression of a new Zac1 transcript we named Zac1o [11]. Our transcriptome assembly validated the existence of Zaclo, and also revealed the presence of another, apparently non-coding transcript sharing the Zac1o CGI as promoter, but transcribed in the opposite direction, a transcript we named Zac1oAS ("AS" for antisense; Fig. 4a). Strikingly, a HyperD overlaps nicely with the oocyte Zac1 transcription unit, which is particularly apparent at the 3' end, where the HyperD and Zac1o transcription unit terminate at essentially the same genomic location (Fig. 4a). We generated a conditional knockout of the Zac1o promoter, resulting in the loss of expression of Zaclo and ZacloAS in oocytes when crossed with the female germline specific CRE deleter transgenic line $\mathrm{Zp3}$-Cre (Figure S7a, b in Additional file 1). As expected from the predictions of our transcription-based model, we found that DNAme fails to be established at the Zac1 igDMR in the absence of transcription, and this loss of methylation is consistent across littermates and litters (Fig. 4b; Figure S7c in Additional file 1). Importantly, this was also the case for the majority of the gene body CpGs we tested, not just within the igDMR (Fig. 4b).

While the hypomethylated state of the Zac1 igDMR is maintained after fertilisation and throughout development in embryos produced from Zac1o-deleted oocytes, CpGs located within the Zaclo gene body but outside the igDMR regained methylation, most likely following embryonic implantation [41] (Fig. 4c). Of interest, deletion 


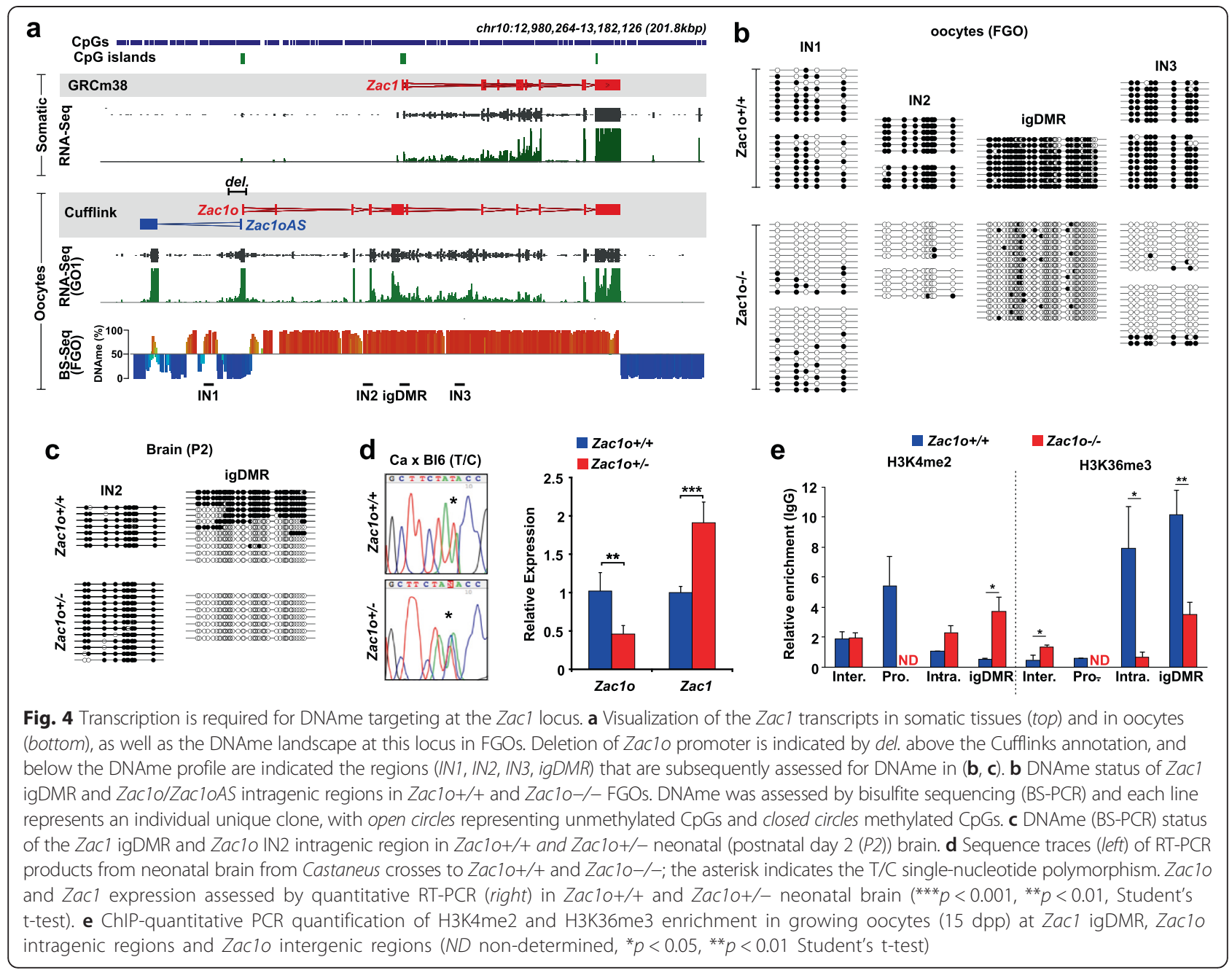

in early embryos of the promoter regulating Zaclo transcription using Sox2-Cre mice did not affect methylation of the igDMR, in accordance with the nature of genomic imprinting, and revealing that transcription is not required for the maintenance of imprints (at least at Zac1) (Figure S7d in Additional file 1). Loss of methylation at the Zac1 igDMR is functionally significant, since it is associated with twofold up-regulation and bi-allelic expression of Zac1 transcripts in all tissues tested, assessed by crossing Zaclo-/- females with Mus castaneus males (Fig. 4d). To validate further this imprinting defect, we also examined the chromatin signature of the Zac1 igDMR by performing ChIP analyses in $\mathrm{Zaclo}+/+$ and $\mathrm{Zaclo}+$ - (maternal deletion in oocytes) embryos (Figure S7e in Additional file 1). This revealed that in embryos inheriting the Zaclo deletion from the oocyte, both parental alleles of the igDMR adopt a paternal epigenotype. Specifically, we noted a loss of the characteristic igDMR allele-specific histone modification signature: there was gain of $\mathrm{H} 3 \mathrm{~K} 4 \mathrm{me} 3$ and $\mathrm{H} 3 \mathrm{~K} 9 \mathrm{ac}$ on the maternal allele, marks normally enriched on the unmethylated paternal allele in $\mathrm{Zaclo}+/+$ embryos, and a marked decrease of the repressive H3K9me3 and H4K20me3, both enriched on the methylated maternal allele in $\mathrm{Zaclo}+/+$ embryos. This observation is reminiscent of those made in embryos 9.5 days post coitum derived from Dnmt3L-/- females that lack female germlinederived DNAme [42].

\section{Transcription is required for full chromatin remodelling at} the Zac1 igDMR

Using our Zac1o mouse model, we sought to investigate in more detail components of the mechanism linking transcription and DNAme. Aside from the role of H3K36me3 in promoting DNMT3A activity, transcription could be implicated in chromatin remodelling at CGIs, ensuring that protective marks are erased [2]. Importantly, the chromatin modifier H3K4me2 demethylase KDM1B has been implicated in DNAme establishment at the Zac1 igDMR [43], and this prompted us to investigate a potential link between KDM1B and transcription. For this we optimised a ChIP-quantitative PCR assay based on 
a previously published micro-ChIP protocol [44]. We prepared chromatin from $\sim 2000$ growing oocytes (15 dpp) for each genotype (Zaclo+/+ and Zaclo-/-), performed immunoprecipitation followed by whole genome amplification in duplicate with multiple quantitative PCR assays per genomic location (intergenic, intragenic, igDMR). To test the accuracy of our protocol, we investigated neutral loci expressed or not in oocytes (Zp3, Ppia, Fam164b; Figure S7f in Additional file 1). Focusing on the Zac1 locus, and as mentioned above, in the absence of Zaclo transcription we found a significant decrease in H3K36me3 within the Zac1o gene body and igDMR. Importantly, we also found that H3K4me2 levels at the igDMR were significantly higher in Zac1o-/- oocytes versus Zac1o+/+ oocytes (Fig. 4e). This result strongly suggests a connection between transcription and recruitment of KDM1B at the Zac1 igDMR, and ultimately that transcription could be implicated in different levels of chromatin remodelling. Of relevance, it was recently reported that human KDM1B binding is enriched in active gene bodies, and it co-precipitates with elongating RNA polymerase II and other transcription elongation factors [45]. Development of reliable ChIP-Seq methods for low cell numbers will allow the connection between transcription and KDM1B at other igDMRs to be investigated in more detail; in addition, it will be important to address more widely the dependence on KDM1B of CGIs methylated in oocytes.

\section{Conclusions}

Our work reveals that the real oocyte transcriptome is only approximated by the publicly available reference annotations. Indeed, we identified thousands of novel genes, coding or non-coding and, in particular, we discovered that many of these transcripts are linked to the de-repression and high expression of TEs from the MaLR and ERVK families in oocytes. Importantly, our transcriptome assembly can be used as a general resource for other scientists and developmental biology questions.

With this transcriptome assembly, we determined that transcription events could account for 85-90\% of DNAme established in the oocyte, including methylated CGIs and igDMRs; however, a small number of expressed genes escape DNAme, as well as a small number of CGIs within active transcription units. By establishing a tight genome-wide correlation between DNAme and active transcription units, as well as functionally demonstrating this at specific genomic loci, our work has wider implications. Indeed, it suggests that gene expression perturbations during oocyte follicular growth could result in alterations in DNAme in mature gametes, including at CGIs. Since a fraction of the oocyte DNA methylome is maintained to some extent in pre-implantation embryos just before the embryonic onset of de novo methylation (the biological consequence of this remains unclear), environmentally induced changes in gene expression in female germ cells could lead to alterations in the epigenome of the next generation, with possible transgenerational effects $[5,8]$.

It is difficult to precisely dissect the mechanisms by which transcription promotes DNAme establishment in oocytes due to the difficulty in obtaining large numbers of these cells. Nevertheless, in vitro biochemical evidence demonstrating a role for H3K36me3 in promoting DNMT3A catalytic activity is obviously central to our understanding, especially since DNMT3B is not active in oocytes. However, and surprisingly given our findings, recent work in mouse ESCs, derived neuronal progenitors, and the human colorectal carcinoma HCT116 cell line have showed that it is principally DNMT3B (and to a lesser extent DNMT3A) that is associated with genebody methylation [46, 47]. Elucidating how DNMT3A specificity towards certain genomic features varies depending on the cellular context would be important to understand DNAme dynamics during early embryonic development and germ cell specification.

By revealing that H3K4me2 removal from the Zac1 igDMR is impaired in the absence of transcription, our results suggest that the role of transcription in DNAme targeting is likely more complex than a simple interaction of DNMT3A with H3K36me3, and could involve the recruitment of histone remodellers or modifiers required for DNAme establishment. Transcription could also indirectly promote complete DNAme establishment by promoting nucleosome displacement, thus ensuring that all CpGs of a given locus can be accessed by the large DNMT3A/3L protein complex, and this is particularly relevant since growing oocytes are in meiotic arrest and not dividing [2].

\section{Materials and methods}

\section{Mouse experimental procedures}

All experimental procedures were approved by the Animal Welfare and Ethical Review Body at the Babraham Institute and were performed under licenses issued by the Home Office (UK) in accordance with the Animals (Scientific Procedures) Act 1986.

\section{RNA-Seq library preparation}

Oocytes were collected from 5-20-day-old C57BL/6Babr mice and RNA was extracted using TRIsure reagent (Bioline) followed by RNA Clean \& Concentrator (Zymo Research) with on-column DNAse treatment (RNasefree DNase I, Life Technologies). Ribosomal RNA was depleted from total RNA using Ribo-Zero Magnetic Kit (Human/Mouse/Rat - Low Input, Epicentre). Libraries 
from GO1 and GO2 were prepared using ScriptSeq v.2 RNA-Seq Library Preparation Kit (Epicentre). To generate sequencing libraries from NGOs and FGOs reverse transcription was performed using SuperScript III (Life Technologies), followed by second DNA strand synthesis using dUTPs instead of dTTPs and DNA polymerase I (NEB); libraries were constructed using the NEBNext DNA Library Prep Master Mix Set for Illumina (NEB), including dUTP excision step by USER Enzyme (NEB) before PCR.

\section{Library sequencing and mapping}

NGO, GO1, GO2 and FGO RNA-Seq libraries were sequenced with 100-bp paired-end reads on an Illumina HiSeq1000. Raw reads were trimmed to remove both poor quality calls and adapters using TrimGalore v.0.2.8 and mapped to the mouse genome (GRCm38 assembly) using TopHat v.2.0.9 (option -g 1). Published RNA-Seq datasets (Table $\mathrm{S} 2$ in Additional file 2) were re-mapped using the same approach in conjunction with gene models from Ensembl release 70, except for the Park et al. dataset, for which TopHat v.2.0. 9 (options - color - quals -g 1) was used. Mapping of reads to repetitive elements is described in Additional file 3.

BS-Seq published datasets (Table S2 in Additional file 2) were trimmed using TrimGalore v.0.2.7 with default parameters, aligned to the mouse genome GRCm38 assembly using Bismark v.0.10.1 (options -pbat, -phred33quals) [48]. CpG methylation calls were extracted from the deduplicated mapping output ignoring the first 4 bp of each read (for post-bisulfite adaptor tagging (PBAT) libraries with $4 \mathrm{~N}$ adapters) using the Bismark methylation extractor (v0.10.0; -no_overlap -report -ignore 4 -ignore_r2 4 for paired-end mode; -report -ignore 4 for the single-end mode).

\section{Oocyte transcriptome assembly}

Transcriptome was assembled using Cufflinks v.2.1.1 $[23,24]$ with default parameters (genome-guided Cufflinks) on a single dataset created by remapping NGO, GO1, GO2, and FGO RNA-Seq datasets (no gene model specification and merging using SAMtools v.0.1.18) and as RABT assembly [25] (option -g) on individual NGO, GO1, GO2, and FGO and merged GO1 and GO2 datasets. For the RABT output, threshold FPKM values to filter non-transcribed transcripts were determined as the point of maximum difference between the values of cumulative distributions of FPKM values of transcripts in the annotation and of random size-matched intergenic regions using a custom $\mathrm{R}$ script. FPKM values were determined using Cufflinks v.2.1.1 with -G option. Transcripts that did not exceed the threshold FPKM were removed. Annotation from genome-guided Cufflinks and filtered annotations from RABT assembler were merged into a single annotation by Cuffmerge.
Potential artefacts in the assembly were detected by visual inspection. Modifications of the annotation GTF file were performed using custom Perl and Java scripts available on request. More details about identification and assessment of the artefacts in the assembly are in Additional file 3.

\section{Curation of the raw Cufflinks annotation}

First, transcripts present in the individual datasets but omitted by Cuffmerge were re-integrated. In some instances, reference transcripts were wrongly assigned FPKM values of 0 by Cufflinks, and re-quantifying the expression of these genes independently led to an increase in almost 2000 predominantly multi-exonic transcripts. In addition, a large number of mono-exonic transcripts $(48,232)$ were found in the raw oocyte annotation, suggesting that some of them could be artefacts; therefore, we applied more stringent criteria for this category. For instance, we removed transcripts without clear directionality information, and transcripts located in introns of multiexonic genes with the same strand orientation that could correspond to remnants of nascent transcripts. We removed mono-exonic transcripts wrongly defined because of issues with the read aligner TopHat (in which a read can be aligned to multiple positions with the same mapping score). We also observed numerous mono-exonic transcripts of the same directionality grouped in clusters, and these were frequently found 3' of multi-exonic transcripts. Since these arrays could result from the incomplete annotation of single longer genes or extended multiexonic transcripts, we merged those transcripts present within a 2-kbp genomic interval of a $3^{\prime}$ end (after having tested multiple size windows and assuming that, theoretically, the number of independent mono-exonic genes on the same strand and on the opposite strand $3^{\prime}$ to a gene should be the same). Finally, since mono-exonic genes can be small, their FPKM values can be relatively high, resulting in artefacts caused by the background noise in RNASeq datasets. We therefore re-quantified mono-exonic genes based solely on read count, and removed lowexpressed ones based on cutoffs determined using normalised random intergenic regions. By performing these corrections on the raw Cufflinks output, the number of multi-exonic transcripts was increased from 65,334 to 67,112 and the number of mono-exonic transcripts was decreased from 48,232 to 15,827 . Of note, the majority of removed transcripts were shorter than $1 \mathrm{kbp}$, while the additional transcripts recovered were predominantly longer than $5 \mathrm{kbp}$ (Fig. 1a; Figure S1c in Additional file 1). The output of our Cufflinks assembly and curation is presented as an annotation track (.gtf file) in Additional file 5.

\section{Transcriptome-related bioinformatic analyses}

The reference transcriptome used in this study was generated using Cuffmerge (Cufflinks v.2.1.1) by merging 
Ensembl, UCSC and RefSeq gene models downloaded from UCSC Table Browser as available on 1 October 2014. Genes were defined as in Cuffcompare within Cufflinks v.2.1.1 output. Oocyte gene coordinates were defined as the most upstream start and the most downstream end coordinates from all transcripts per gene. Transcripts were categorised into reference and novel by Cuffcompare, with categories $=, \mathrm{c}, \mathrm{j}$, and o marking the transcripts of reference genes and categories $\mathrm{i}, \mathrm{u}$, and $\mathrm{x}$ novel transcripts.

CGIs and igDMRs were defined as published [7, 9, 49, 50] and lifted over using the UCSC liftover tool into the GRCm38 assembly, removing CGIs on Y chromosome. CGIs were classified as TSS-associated if they overlap the most upstream TSS of a gene $\pm 100 \mathrm{bp}$, intragenic if they overlap the gene but are not at the TSS, and intergenic without gene overlap. Coordinates for TEs (L1 and L2 LINEs, S2 and S4 SINEs, ERV1s, ERVKs, ERVLs, MaLRs) for the mouse GRCm38 genome build were generated using RepeatMasker. TSSs were classified as CGI-associated if a first base pair of a gene or transcript \pm 100 -bp overlapped a CGI and as TEassociated if a first base pair of a gene or transcript overlapped a TE on the same strand.

Expression of assembled transcripts in published oocyte, embryonic, and differentiated cell datasets (Table S2 in Additional file 2) was quantified using Cufflinks v.2.1.1 (option-G). Expression of genes was determined as a sum of FPKM values of all transcripts per gene. Expression levels in individual embryonic datasets (single cells) were merged per stage taking the total read count in each dataset into consideration. Expression of upstream and reference TSSs at each stage or cell type was estimated in Seqmonk for exons containing upstream or reference TSSs as read count quantification corrected for length and then manually corrected for read count in individual or merged datasets to obtain RPKM values.

To perform hierarchical clustering, only genes with a FPKM value of at least 0.1 in at least one dataset were selected. Log transformed values were mean-centred and clustered based on Pearson's correlation using the hclust function in R v.3.0.2. All statistical analyses (chi-squared tests) were performed in $\mathrm{R}$ v.3.0.2.

\section{Genome-wide DNA methylation analysis}

To define hyper- and hypomethylated domains (HyperD, HypoD), probes were designed over $\mathrm{CpGs}$ with data [35], merging 50 consecutive CpGs with step size of ten CpGs. Methylation percentage level was then quantified taking into account only CpGs covered by at least five reads and a minimum of three positions to count a probe. Exported data were then processed using custom Perl scripts (available on request) as shown in Figure S4 in Additional file 1. Overlapping windows with methylation level $>75 \%$ and $<25 \%$ were merged into HyperDs and HypoDs, respectively, splitting overlapped regions between HyperDs and HypoDs into halves. Then, neighbouring domains of the same status were merged if a gap between them was $<2 \mathrm{kbp}$, or if there was a small domain $(<1 \mathrm{kbp})$ of the opposite status between them. Small domains $(<2 \mathrm{kpb})$ were then removed and, again, neighbouring domains of the same status were merged if a gap between them was $<2 \mathrm{kbp}$.

For correlation with the transcriptome, CGIs, TSSs, and 1-kbp regions (three CpGs with at least three reads) with $\geq 50 \%$ methylation in NGOs or DNMT3Aor DNMT3L-deficient oocytes were excluded from the domains using a custom Perl script. TSSs excluded from the domain designation were defined as $2-\mathrm{kbp}$ regions downstream of a gene's most upstream TSS. If a domain was divided into more parts, the information about the parental domain was preserved for adequate correlation with transcription and other features.

Oocyte contigs were defined as genomic regions with three or more reads on the same strand in at least one of the oocyte datasets. Enrichment in ERVK elements and in intermediate levels of methylation (25-50\%) in NGOs or DNMT3A- and DNMT3L-deficient oocytes was quantified by the comparison of numbers of nontranscribed ( $<25 \%$ overlap by transcripts) HyperDs and HypoDs with $>50 \%$ overlap with these features, requiring $p$ value $<0.0001$ in chi-squared test.

A FPKM threshold of 0.5 for gene bodies remaining unmethylated was defined by quantification of the proportion of unmethylated gene bodies from all gene bodies with increasing FPKM values $(0-0.1,0.1-0.2$, $0.2-0.3$, etc.). Below a FPKM of 0.5, more genes were unmethylated than methylated. CpG density and GC content were quantified using a custom Perl script from GRCm38 genome assembly. All methylation levels were quantified in Seqmonk, using the following parameters: three CpGs with a minimum of three reads depth to count a probe for gene bodies; ten CpGs with a minimum of five reads depth for CGIs; a minimum of five reads depth for individual CpGs. Statistical analyses were performed in $\mathrm{R}$ v.3.0.2.

\section{Generation of Zac1o conditional deletion mice}

The targeting construct was prepared using homologous recombination in bacteria. We inserted one loxP site upstream (2.6 kbp) of the Zac1o first exon and one loxP downstream together with a neomycin selection cassette flanked by Frt sites. The targeting construct was electroporated in C57BL/6J Bruce4 ESCs, and correct integration assessed by Southern blot. Chimeric mice were generated by injecting targeted ESCs into C57BL/6J blastocysts and crossed with female Flpe-Cre mice for excision of the selection cassette. Specific deletion of 
the Zac1o first exon and promoter in oocytes was performed by crossing with $\mathrm{Zp3}$-Cre mice. For experiments with allelic information, Zaclo-floxed or Zac1o-deleted female mice were crossed with $M$. castaneus wild-type males (CAST/EiJ).

\section{Bisulfite-PCR sequencing and COBRA analysis}

Oocytes were collected by mouth pipetting as previously described [11], and lysed at $37^{\circ} \mathrm{C}$ for $1 \mathrm{~h}$ (SDS $0.5 \%$ final, EDTA $0.5 \mathrm{mM}$ final, phosphate-buffered saline, $10 \mu \mathrm{g}$ of proteinase K). Bisulfite conversion was performed directly on cell lysates. For tissues, DNA was first purified using phenol-chloroform extraction, $500 \mathrm{ng}$ used for bisulfite conversion, and $50 \mathrm{ng}$ equivalent in each PCR reaction. Bisulfite conversion was performed using a commercial kit according to the manufacturer's recommendations (Sigma, Imprint DNA modification kit, two-step protocol). PCR was performed using Pfu Turbo Cx Polymerase (Stratagene). Primer sequences are available upon request. Cloning and analysis were performed as described elsewhere [5], with 20-25 clones analysed per genomic region and removal of clones with identical patterns of conversion based on both $\mathrm{CpG}$ and non-CpG methylation. For COBRA analysis, DNA methylation of the Zac1 igDMR was assessed using Taq1 restriction endonuclease.

\section{Chromatin immunoprecipitation in oocytes}

Growing oocytes were collected from 15-dpp females as previously described [11], fixed at room temperature in 4 $\%$ formaldehyde for $15 \mathrm{~min}$, washed in phosphatebuffered saline with a final wash in less than $5 \mu \mathrm{l}$, snapfrozen and stored at $-80{ }^{\circ} \mathrm{C}$ before lysis. In total, 2180 Zac1o-deleted and 1975 wild-type oocytes were processed. Lysis and immunoprecipitation were performed using the True MicroChIP kit (Diagenode AB-002-0016) with the following modifications. Aliquoted oocytes were lysed using $50 \mu \mathrm{l}$ total lysis buffer tL1 and incubated on ice for $10 \mathrm{~min}$. Equivalent of $150 \mu \mathrm{l}$ of ice-cold HBBS buffer was added and all lysates were pooled together in 1.5 $\mathrm{ml}$ TPX microtubes (Diagenode). Chromatin shearing was performed using the Bioruptor (Diagenode) with five active cycles (30 s ON, $30 \mathrm{~s}$ OFF). Tubes were centrifuged at $14,000 \mathrm{~g}$ for $15 \mathrm{~min}$ at $4{ }^{\circ} \mathrm{C}$ and supernatant collected in a 1.5-ml low-binding tube. Ice-cold complete ChIP buffer tC1 $(200 \mu \mathrm{l})$ was added, and the total volume was divided in three, equally. H3K36me3 (0.25 $\mu \mathrm{g}$; Active Motif, 61102), $0.5 \mu \mathrm{g}$ of H3K4me2 (Abcam, ab32356) and $0.25 \mu$ g of IgG (Abcam, ab46540) antibodies were used per immunoprecipitation according to the manufacturers' protocols, except that DNA purification following removal of cross-links was performed using AMPure XP beads (1.8× ratio, Agencourt). Immunoprecipitated material was separated in two equally, and whole-genome amplification was performed according to the manufacturer's protocol (WGA4, Sigma-Aldrich, starting from step 6) for nine cycles. We subsequently submitted $1 \mu \mathrm{l}$ to 15 additional amplification cycles for agarose gel visualisation purposes. The remaining amplified material was purified using AMPure XP beads according to the manufacturer's recommendations (1.8 $\times$ ratio, Agencourt), and quantitative PCR performed, with quantification as relative enrichment to IgG and correction for primer efficiency. For $Z p 3$, two independent PCR assays were designed for intergenic surrounded regions, two for the promoter region, and three for the gene body; for Ppia, this was two intergenic, two promoter, and four intragenic regions; for Fam164b, this was two intergenic, two promoter, and three intragenic regions; for the Zac1/Zaclo regions, this was two independent assays for intergenic regions, two for the Zaclo promoter, three for Zac1o intragenic regions, and three for the Zac1 igDMR. All primer sequences are available upon request.

\section{Chromatin immunoprecipitation in embryos}

ChIP of native chromatin was carried out as described previously [42]. Three ChIP assays were performed using independent chromatin preparations, with anti-H3K4me3 (Diagenode pAb 030-050), anti-H3K9ac (Merck-Millipore 06-942), anti-H3K9me3 (Merck-Millipore 07-442) and anti-H4K20me3 (Merck-Millipore 07-463). Analysis of immunoprecipitated chromatin was done as follows: in the input and antibody-bound fractions for each antiserum used, the parental alleles were differentiated by direct sequencing of the PCR products encompassing a strainspecific single-nucleotide polymorphism in the regions of interest. Input and antibody-bound fractions were quantified by real-time PCR amplification with a SYBR Green mixture (Roche) using a LightCycler ${ }^{\circledR} 480 \mathrm{II}$ (Roche) instrument. Background precipitation levels were determined by performing mock precipitations with a non-specific IgG antiserum (Sigma C-2288) and were only a fraction of the precipitation levels obtained with specific antisera. Bound/ input ratios were calculated and normalised to those for the imprinted $K v D M R$, which we showed to be similar in wild-type and mutant embryos.

\section{Data availability}

The datasets supporting the results of this article are available in the Gene Expression Omnibus repository, under accession number [GEO:GSE70116].

\section{Additional files}

Additional file 1: Supplementary Figures S1-S7 with their legends. (PDF $5482 \mathrm{~kb}$ ) 
Additional file 2: Supplementary Tables S1-S4. (PDF $240 \mathrm{~kb}$ ) Additional file 3: Supplementary methods, including more details on the transcriptome assembly process. (PDF $107 \mathrm{~kb}$ )

Additional file 4: A table with the genomic coordinates of our final definition of HyperDs and HypoDs (GRCm38 assembly). (XLSX $4255 \mathrm{~kb}$ ) Additional file 5: A gtf file corresponding to the annotation of oocyte transcripts according to our transcriptome assembly (GRCm38 assembly; this version does not include expressed independent repetitive elements). (GTF $50970 \mathrm{~kb}$ )

\section{Abbreviations}

bp: base pair; BS: bisulfite sequencing; CGI: CpG island; ChIP: chromatin immunoprecipitation; CNCl: Coding-Non-Coding Index; CPC: Coding Potential Calculator; DNAme: DNA methylation; DNMT: DNA methyltransferase; dpp: days post-partum; E: embryonic day; ESC: embryonic stem cell; FGO: fully grown oocyte; FPKM: fragments per kilobase of transcript per million mapped reads; GO: growing oocyte; HyperD: hypermethylated domain; HypoD: hypomethylated domain; igDMR: imprinted germline differentially methylated regions; ncRNA: non-coding RNA; NGO: non-growing oocyte; PCR: polymerase chain reaction; PGC: primordial germ cell; RABT: reference annotation-based transcript; RNA-Seq: RNA sequencing; RRBS: reduced representation bisulfite sequencing; TE: transposable element; TSS: transcription start site; UCSC: University of California, Santa Cruz.

\section{Competing interests}

The authors declare that they have no competing interests.

\section{Authors' contributions}

LV performed RNA-Seq, transcriptome assembly, and global correlation with transcription. SAS. generated Zaclo conditional knockout mice, and performed Zac1o-related experiments except ChIP in embryos, which was performed by SM-M, and PA, LV, HS, FK, and SA performed bioinformatic analyses. KS and ST provided material and advice. GK and SAS initiated and supervised the project. SAS wrote the manuscript with input from LV and GK. All authors read and approved the final manuscript.

\section{Acknowledgements}

We thank all members of the Kelsey laboratory and Wendy Dean for helpful discussions, Kristina Tabbada for assistance with Illumina sequencing, and Solenn Patalano for help with RNA-Seq methodology. This work was supported by MRC Programme grant MR/K011332/1, MRC Project grant G0800013, FP6 SAVEBETA, and a Babraham Institute and University of Cambridge graduate student scholarship.

\section{Author details}

'Epigenetics Programme, Babraham Institute, Cambridge, UK. ${ }^{2}$ Bioinformatics Group, Babraham Institute, Cambridge, UK. ${ }^{3} \mathrm{GReD}, \mathrm{CNRS}$, INSERM, and Clermont University, 63001 Clermont-Ferrand, France. ${ }^{4}$ Department of Histology and Cell Biology, Yokohama City University School of Medicine, Yokohama, Japan. ${ }^{5}$ Centre for Trophoblast Research, University of Cambridge, Cambridge, UK.

Received: 24 June 2015 Accepted: 3 September 2015

Published online: 25 September 2015

\section{References}

1. Seisenberger S, Peat JR, Hore TA, Santos F, Dean W, Reik W. Reprogramming DNA methylation in the mammalian life cycle: building and breaking epigenetic barriers. Philos Trans R Soc Lond B Biol Sci. 2012;368:20110330-0.

2. Smallwood SA, Kelsey G. De novo DNA methylation: a germ cell perspective. Trends Genet. 2012;28:33-42.

3. Ferguson-Smith AC. Genomic imprinting: the emergence of an epigenetic paradigm. Nat Rev Genet. 2011;12:565-75.

4. Kobayashi H, Sakurai T, Imai M, Takahashi N, Fukuda A, Yayoi O, et al. Contribution of intragenic DNA methylation in mouse gametic DNA methylomes to establish oocyte-specific heritable marks. PLoS Genet. 2012;8:e1002440. doi:10.1371/journal.pgen.1002440.
5. Smallwood SA, Tomizawa S-I, Krueger F, Ruf N, Carli N, Segonds-Pichon A, et al. Dynamic CpG island methylation landscape in oocytes and preimplantation embryos. Nat Genet. 2011;43:811-4.

6. Smith ZD, Chan MM, Mikkelsen TS, Gu H, Gnirke A, Regev A, et al. A unique regulatory phase of DNA methylation in the early mammalian embryo. Nature. 2012;484:339-44.

7. Wang L, Zhang J, Duan J, Gao X, Zhu W, Lu X, et al. Programming and inheritance of parental DNA methylomes in mammals. Cell. 2014;157:979-91.

8. Borgel J, Borgel J, Guibert S, Guibert S, Li Y, Li Y, et al. Targets and dynamics of promoter DNA methylation during early mouse development. Nat Genet. 2010;42:1093-100.

9. Proudhon C, Duffié R, Ajjan S, Cowley M, Iranzo J, Carbajosa G, et al. Protection against de novo methylation is instrumental in maintaining parent-of-origin methylation inherited from the gametes. Mol Cell. 2012:47:909-20.

10. Messerschmidt DM, Knowles BB, Solter D. DNA methylation dynamics during epigenetic reprogramming in the germline and preimplantation embryos. Genes Dev. 2014;28:812-28.

11. Chotalia M, Smallwood SA, Ruf N, Dawson C, Lucifero D, Frontera M, et al. Transcription is required for establishment of germline methylation marks at imprinted genes. Genes Dev. 2009;23:105-17.

12. Fröhlich LF, Mrakovcic M, Steinborn R, Chung U-I, Bastepe M, Jüppner H. Targeted deletion of the Nesp55 DMR defines another Gnas imprinting control region and provides a mouse model of autosomal dominant PHP-Ib. Proc Natl Acad Sci U S A. 2010;107:9275-80.

13. Smith EY, Futtner CR, Chamberlain SJ, Johnstone KA, Resnick JL. Transcription is required to establish maternal imprinting at the Prader-Will syndrome and Angelman syndrome locus. PLoS Genet. 2011;7:e1002422.

14. Dhayalan A, Rajavelu A, Rathert P, Tamas R, Jurkowska RZ, Ragozin S, et al. The Dnmt3a PWWP domain reads histone 3 lysine 36 trimethylation and guides DNA methylation. J Biol Chem. 2010;285:26114-20.

15. Zhang Y, Jurkowska R, Soeroes S, Rajavelu A, Dhayalan A, Bock I, et al. Chromatin methylation activity of Dnmt3a and Dnmt3a/3L is guided by interaction of the ADD domain with the histone $\mathrm{H} 3$ tail. Nucleic Acids Res. 2010;38:4246-53.

16. Park S-J, Komata M, Inoue F, Yamada K, Nakai K, Ohsugi M, et al. Inferring the choreography of parental genomes during fertilization from ultralarge-scale whole-transcriptome analysis. Genes Dev. 2013;27:2736-48.

17. Macfarlan TS, Gifford WD, Driscoll S, Lettieri K, Rowe HM, Bonanomi D, et al. Embryonic stem cell potency fluctuates with endogenous retrovirus activity. Nature. 2012:487:57-63. doi:10.1038/nature11244.

18. Tang F, Barbacioru C, Nordman E, Bao S, Lee C, Wang X, et al. Deterministic and stochastic allele specific gene expression in single mouse blastomeres. PLoS One. 2011;6:e21208. doi:10.1371/journal.pone.0021208.

19. Guttman M, Garber M, Levin JZ, Donaghey J, Robinson J, Adiconis X, et al. Ab initio reconstruction of cell type-specific transcriptomes in mouse reveals the conserved multi-exonic structure of lincRNAs. Nat Biotechnol. 2010;28:503-10.

20. Ramsköld D, Luo S, Wang Y-C, Li R, Deng Q, Faridani OR, et al. Full-length mRNA-Seq from single-cell levels of RNA and individual circulating tumor cells. Nat Biotechnol. 2012;30:777-82.

21. Xue Z, Huang K, Cai C, Cai L, Jiang C-Y, Feng Y, et al. Genetic programs in human and mouse early embryos revealed by single-cell RNA sequencing. Nature. 2013;500:593-7.

22. Derrien T, Johnson R, Bussotti G, Tanzer A, Djebali S, Tilgner $H$, et al. The GENCODE V7 catalog of human long noncoding RNAs: analysis of their gene structure, evolution, and expression. Genome Res. 2012;22:1775-89

23. Trapnell C, Williams BA, Pertea G, Mortazavi A, Kwan G, van Baren MJ, et al. Transcript assembly and quantification by RNA-Seq reveals unannotated transcripts and isoform switching during cell differentiation. Nat Biotechnol. 2010;28:511-5.

24. Trapnell C, Roberts A, Goff L, Pertea G, Kim D, Kelley DR, et al. Differentia gene and transcript expression analysis of RNA-Seq experiments with TopHat and Cufflinks. Nat Protoc. 2012;7:562-78.

25. Roberts A, Pimentel $\mathrm{H}$, Trapnell C, Pachter L. Identification of novel transcripts in annotated genomes using RNA-Seq. Bioinformatics. 2011:27:2325-9.

26. Palmieri N, Nolte V, Suvorov A, Kosiol C, Schlötterer C. Evaluation of different reference based annotation strategies using RNA-Seq - a case study in Drososphila pseudoobscura. PLoS One. 2012;7:e46415. 
27. Xie C, Yuan J, Li H, Li M, Zhao G, Bu D, et al. NONCODEv4: exploring the world of long non-coding RNA genes. Nucleic Acids Res. 2014;42:D98-D103.

28. Kong L, Zhang Y, Ye Z-Q, Liu X-Q, Zhao S-Q, Wei L, et al. CPC: assess the protein-coding potential of transcripts using sequence features and support vector machine. Nucleic Acids Res. 2007;35:W345-9.

29. Sun L, Luo H, Bu D, Zhao G, Yu K, Zhang C, et al. Utilizing sequence intrinsic composition to classify protein-coding and long non-coding transcripts. Nucleic Acids Res. 2013;41:e166-6.

30. Altschul SF, Madden TL, Schäffer AA, Zhang J, Zhang Z, Miller W, et al. Gapped BLAST and PSI-BLAST: a new generation of protein database search programs. Nucleic Acids Res. 1997;25:3389-402.

31. Shin Y-H, Choi Y, Erdin SU, Yatsenko SA, Kloc M, Yang F, et al. Hormad1 mutation disrupts synaptonemal complex formation, recombination, and chromosome segregation in mammalian meiosis. PLoS Genet. 2010;6:e1001190

32. Flemr M, Malik R, Franke V, Nejepinska J, Sedlacek R, Vlahovicek K, et al. A retrotransposon-driven dicer isoform directs endogenous small interfering RNA production in mouse oocytes. Cell. 2013;155:807-16.

33. Peaston $A E$, Evsikov AV, Graber $J H$, de Vries WN, Holbrook AE, Solter D, et al. Retrotransposons regulate host genes in mouse oocytes and preimplantation embryos. Dev Cell. 2004;7:597-606.

34. Fadloun A, Le Gras S, Jost B, Ziegler-Birling C, Takahashi H, Gorab E, et al Chromatin signatures and retrotransposon profiling in mouse embryos reveal regulation of LINE-1 by RNA. Nat Struct Mol Biol. 2013;20:332-8.

35. Shirane K, Toh H, Kobayashi H, Miura F, Chiba H, Ito T, et al. Mouse oocyte methylomes at base resolution reveal genome-wide accumulation of nonCpG methylation and role of DNA methyltransferases. PLoS Genet. 2013;9:e1003439.

36. Rebollo R, Miceli-Royer K, Zhang Y, Farivar S, Gagnier L, Mager DL. Epigenetic interplay between mouse endogenous retroviruses and host genes. Genome Biol. 2012;13:R89.

37. Li B, Gogol M, Carey M, Pattenden SG, Seidel C, Workman JL. Infrequently transcribed long genes depend on the Set2/Rpd3S pathway for accurate transcription. Genes Dev. 2007:21:1422-30

38. Clouaire T, Webb S, Skene P, Illingworth R, Kerr A, Andrews R, et al. Cfp1 integrates both $\mathrm{CpG}$ content and gene activity for accurate H3K4me3 deposition in embryonic stem cells. Genes Dev. 2012;26:1714-28.

39. Stadler MB, Murr R, Burger L, Ivanek R, Lienert F, Schöler A, et al. DNAbinding factors shape the mouse methylome at distal regulatory regions. Nature. 2011:480:490-5. doi:10.1038/nature10716.

40. Docherty LE, Kabwama S, Lehmann A, Hawke E, Harrison L, Flanagan SE, et al. Clinical presentation of 6q24 transient neonatal diabetes mellitus (6q24 TNDM) and genotype-phenotype correlation in an international cohort of patients. Diabetologia. 2013;56:758-62.

41. Auclair G, Guibert S, Bender A, Weber M. Ontogeny of CpG island methylation and specificity of DNMT3 methyltransferases during embryonic development in the mouse. Genome Biol. 2014;15:545.

42. Henckel A, Nakabayashi K, Sanz LA, Feil R, Hata K, Arnaud P. Histone methylation is mechanistically linked to DNA methylation at imprinting control regions in mammals. Hum Mol Genet. 2009;18:3375-83.

43. Ciccone DN, Su H, Hevi S, Gay F, Lei H, Bajko J, et al. KDM1B is a histone H3K4 demethylase required to establish maternal genomic imprints. Nature. 2009;461:415-8.

44. Dahl JA, Reiner AH, Collas P. Fast genomic $\mu$ ChIP-chip from 1,000 cells. Genome Biol. 2009:10:R13.

45. Fang R, Barbera AJ, Xu Y, Rutenberg M, Leonor T, Bi Q, et al. Human LSD2/ KDM1b/AOF1 regulates gene transcription by modulating intragenic H3K4me2 methylation. Mol Cell. 2010;39:222-33.

46. Baubec T, Colombo DF, Wirbelauer C, Schmidt J, Burger L, Krebs AR, et al. Genomic profiling of DNA methyltransferases reveals a role for DNMT3B in genic methylation. Nature. 2015;520:243-7.

47. Yang X, Han H, De Carvalho DD, Lay FD, Jones PA, Liang G. Gene body methylation can alter gene expression and is a therapeutic target in cancer. Cancer Cell. 2014:26:577-90.

48. Krueger F, Andrews SR. Bismark: a flexible aligner and methylation caller for Bisulfite-Seq applications. Bioinformatics. 2011:27:1571-2.
49. Illingworth RS, Gruenewald-Schneider U, Webb S, Kerr ARW, James KD, Turner DJ, et al. Orphan CpG islands identify numerous conserved promoters in the mammalian genome. PLoS Genet. 2010;6:e1001134.

50. Tomizawa S-I, Kobayashi H, Watanabe T, Andrews S, Hata K, Kelsey G, et al. Dynamic stage-specific changes in imprinted differentially methylated regions during early mammalian development and prevalence of non-CpG methylation in oocytes. Development. 2011;138:811-20.

\section{Submit your next manuscript to BioMed Central and take full advantage of:}

- Convenient online submission

- Thorough peer review

- No space constraints or color figure charges

- Immediate publication on acceptance

- Inclusion in PubMed, CAS, Scopus and Google Scholar

- Research which is freely available for redistribution 